\title{
Trivium
}

Revue franco-allemande de sciences humaines et sociales - Deutsch-französische Zeitschrift für Geistesund Sozialwissenschaften

28 | 2018

Capitalisme

\section{Unternehmensgeschichte und Kapitalismus}

\section{Patrick Fridenson}

Traducteur : Gisela Naegle

\section{OpenEdition}

Journals

Édition électronique

URL : http://journals.openedition.org/trivium/5993

DOI : 10.4000/trivium.5993

ISBN : 1963-1820

ISSN : $1963-1820$

Éditeur

Les éditions de la Maison des sciences de l'Homme

\section{Référence électronique}

Patrick Fridenson, « Unternehmensgeschichte und Kapitalismus », Trivium [Online], 28 | 2018, online erschienen am 26 November 2018, abgerufen am 08 September 2020. URL : http:// journals.openedition.org/trivium/5993 ; DOI : https://doi.org/10.4000/trivium.5993

Ce document a été généré automatiquement le 8 septembre 2020.

\section{(c) () (9)}

Les contenus des la revue Trivium sont mis à disposition selon les termes de la Licence Creative Commons Attribution - Pas d'Utilisation Commerciale - Pas de Modification 4.0 International. 


\section{Unternehmensgeschichte und Kapitalismus}

\section{Patrick Fridenson}

Traduction : Gisela Naegle

1 Es ist inzwischen allgemein bekannt, dass das Wort "Kapitalismus" zuerst in der französischen Sprache gebraucht wurde, und zwar von den Sozialisten Louis Blanc (1850) und Pierre-Joseph Proudhon (1851). (1867 wurde es in dieser Sprache von Pierre Larousse mit dem Sinn in das Grand Dictionnaire Universel du XIXe siècle aufgenommen, den Proudhon ihm gegeben hatte, der »Macht des Kapitals oder der Kapitalisten«). ${ }^{1}$ Die Sozialwissenschaften verschiedener Länder zögerten nicht, diese Begrifflichkeit aufzugreifen. In Deutschland und Frankreich kann man dabei insbesondere auf zwei Gruppen von Arbeiten verweisen, die, jeweils um die Jahrhundertwende, bahnbrechend waren - wenn auch in ganz unterschiedlichen Formen. 1902 publizierte der Ökonom Werner Sombart eine Gesamtdarstellung in zwei Bänden: Der moderne Kapitalismus. Historisch-systematische Darstellung des gesamteuropäischen Wirtschaftslebens von seinen Anfängen bis zur Gegenwart, ${ }^{2}$ in der er die Anfänge des Systems charakterisierte. Die Ambitionen des französischen Wirtschaftshistorikers Henri Hauser waren weitaus bescheidener, als er, ebenfalls noch im Jahr 1902, in der Revue d'économie politique einen 35-seitigen Artikel über die Anfänge des Kapitalismus in Frankreich veröffentlichte, der drei im April 1901 gehaltene Vorträge aufnahm. Beide Publikationen stimmten darin überein, dass sie einen Zeitraum behandelten, der vom Ende des Mittelalters bis in das frühe 18. Jahrhundert reichte.

Diese beiden Veröffentlichungen wurden überarbeitet und erweitert. Sombart gab sich nicht damit zufrieden, seinen Text 1916 substanziell zu verändern, sondern er fügte ihm 1928 zwei weitere Bände hinzu, die die Periode von der Industriellen Revolution bis 1914 behandelten. 1927 vereinigte Hauser seinen Artikel von 1902 und sieben spätere Artikel zu einem Buch und stellte ihnen eine allgemeine Einführung voran. ${ }^{3}$ Aber die Unterschiede betrafen nicht nur die Ambitionen. Die Akzentuierung dieser beiden Arbeiten unterschied sich ganz grundsätzlich. So unterstrich Sombart die Rolle des Luxus und des Krieges für das Aufkommen des Kapitalismus, ebenso wie die 
Bedeutung der doppelten Buchführung. Er zeichnete die Entwicklungen im historischen Profil der Unternehmer nach, gleichgültig, ob es sich dabei um Kaufleute, Finanziers oder Industrielle handelte. Für Hauser war das Kapital zu einem "wesentlichen Faktor der industriellen Produktion geworden«, der »einen Anteil von den Profiten der Industrie abschöpft«. Die bevorzugten Untersuchungsgegenstände waren die Technik und die Arbeitsteilung, die Konzentration des Kapitals und die Kämpfe zwischen der Arbeit und dem Kapital.

In beiden Ansätzen war von Kapitalisten und Kapitalien, von Buchhaltungsmethoden, von der Währung und von den Staaten die Rede, aber die Unternehmen als solche spielten keine zentrale Rolle. Dies war charakteristisch für die Wirtschaftsgeschichte, so wie sie seit der Mitte des 19.Jahrhunderts in der gesamten westlichen Welt aufgefasst und praktiziert wurde; in der Zwischenkriegszeit weitete sie ihr Feld auf die Untersuchung von Preisen aus.

4 Was konnte also die neue Disziplin der Unternehmensgeschichte zur Untersuchung des Kapitalismus beitragen? In einem ersten Schritt werden wir zeigen, wie sich die Unternehmensgeschichte außerhalb des regelmäßigen Austauschs unter Historikern konstituierte - und das hauptsächlich als Antwort auf eine Nachfrage von Seiten der Betriebswissenschaften in den USA. In einem zweiten Schritt werden wir erklären, warum Frankreich trotz der Tätigkeit Marc Blochs diese Trendwende zunächst verpasst hat. In einem dritten Schritt werden wir sehen, wie es dazu kam, dass sich seit 1945 die Unternehmensgeschichte in einem weltweiten Maßstab ausbreiten konnte, indem sie anfangs verschiedene intellektuelle Problematiken zusammenfasste und sich danach, in Abhängigkeit vom jeweiligen Land, unterschiedlich weiterentwickelte. Die Analyse des Kapitalismus wurde dadurch bereichert, aber nach der Zeit Alfred Chandlers konnte sich keine weltweit einheitliche Interpretation mehr durchsetzen. In einem letzten Abschnitt skizzieren wir die Hauptthemen, die heute die Geschichte des französischen Kapitalismus charakterisieren.

\section{Eine vielschichtige Entstehung - vor allem in den USA}

5 Die Unternehmensgeschichte erblickte nicht etwa in den ersten beiden großen Industrienationen das Licht der Welt, sondern in zwei jüngeren Industrieländern - und in beiden Fällen außerhalb der universitären Geschichtslehrstühle.

6 Deutschland leistete zwar Pionierarbeit, aber die Initiativen auf dem Gebiet der Unternehmensgeschichte blieben isoliert. Der Ökonom Richard Ehrenberg publizierte 1896 eine große Geschichte der Fugger, einer bedeutenden Bankiersfamilie des 16. Jahrhunderts, dann ein Buch zur Geschichte einer Hamburger Firma, der Parish (1905), und schließlich, 1912 und 1925, zwei Bücher zu dem metallverarbeitenden Betrieb Krupp, seinen Arbeitern und seinen Führungskräften. Der Ingenieur Conrad Matschoss schrieb Geschichten der Firmen Sulzer (1910), R. Wolf (1912), Deutz (1921), Loewe (1930), Bosch (1931) und Wanderer (1935).

7 Als vollwertige Disziplin entstand die Unternehmensgeschichte aber trotzdem in den USA. Man könnte glauben, sie sei aus einer Diversifizierung der Wirtschaftsgeschichte hervorgegangen. Aber das stimmt nicht. Denn es waren die Betriebswissenschaften, die das Bedürfnis nach dieser neuen Disziplin zum Ausdruck brachten und die ihr ihren 
Namen gaben: business history. Der erste Professor, der auf einen Lehrstuhl für Unternehmensgeschichte berufen wurde, Norman S. B. Gras, schrieb, dass die 1925 gegründete Business Historical Society,

»als Teil der Vergrößerung der Harvard Graduate School of Business Administration konzipiert war. Genauer gesagt war sie das Ergebnis der Bemühung, die Forschung und Ausbildung für den Bereich des Geschäftslebens auszudehnen. Aus diesen Bestrebungen sind neue Spezialisierungen innerhalb der Unternehmensgeschichte entstanden, in der Geschäftsethik - Studien, die schon seit langem überfällig waren, Themen, für die es uns möglich wäre, die frei baumelnden Fäden einer kurzsichtigen Vergangenheit zusammenzubinden«.

8 Auf der ersten Seite des Artikels, auf der er die ersten 25 Jahre der Vereinigung nachzeichnete, fügte er drei aufschlussreiche Züge hinzu: »Sie wurde von Männern begründet [...] die ihren Weg in die Richtung eines Ziels lenkten, das sie nur ungefähr erkannt hatten.« Theorie stand nicht auf der Tagesordnung. Es ging darum, empirische Forschungen durchzuführen. Die neue Vereinigung »vergrößerte nicht die Anzahl amerikanischer Vereinigungen, die die Schwächen der Nation ausbreiteten«. Sie gehörte grundsätzlich $\mathrm{zu}$ den ausgedehnten Bemühungen, auf das seit dem 19. Jahrhundert - der »Progressive Era« - bei einem erheblichen Teil der Farmer, der Arbeiter, der Einzelhändler, der Beamten und der Intellektuellen zu beobachtende Aufblühen der Kritik sowohl an Großunternehmen als auch am Image der USA selbst zu reagieren. ${ }^{4}$

Die Konkretisierung dieses Projektes war Wallace B. Donham zu verdanken, einem ehemaligen Bankier, der nach dem Ende des Krieges, 1919, Dekan der Harvard Business School wurde. Er überzeugte sich von der Notwendigkeit, die 1908 gegründete Schule zu verändern. 1922 schuf er ein monatliches Magazin, um dem breiten Publikum der leitenden Angestellten die Forschungsansätze und Überzeugungen der Harvard Business School bekannt zu machen: den Harvard Business Review. 1924 erklärte er, dass die Ausbildung der Führungskräfte mit Hilfe der Einrichtung dreier neuer Ausbildungsgänge vervollkommnet werden sollte: der Unternehmensgeschichte, der Geschäftsethik und der human relations. Er war der Ansicht, dass dieses ehrgeizige Programm das Aufbringen erheblicher Kredite erforderte und es gelang ihm schließlich, dafür eine Spende von 5 Millionen Dollar von George F. Baker, einem großen Depositen-Bankier und bekannten Philanthropen, zu erhalten. Er fand auch intellektuellen Rückhalt in den in Harvard von dem 1924 rekrutierten Philosophen Alfred Whitehead entwickelten Ideen zur Betriebsführungs-Ausbildung. ${ }^{5}$ Sein Projekt zur Unternehmensgeschichte war besonders kohärent: Es beschränkte sich nicht auf die Schaffung eines Lehrangebots, sondern bezweckte auch, Unternehmensarchive zu sammeln und zu erhalten und Forschungen auf diesem neuen Gebiet anzuregen. Dieser ehemalige Bankier, zum Dekan der Betriebswissenschaft geworden, war es also, der den Ausdruck business history prägte und eine Vereinigung schuf, die den Auftrag hatte, die Sammlung von Archiven und die Popularisierung der Disziplin zu fördern. Er rief ihr Organ ins Leben: das Bulletin of the Business Historical Society (1926-1953), das 1954 zum Business History Review wurde. Er war es auch, der auf den Vorschlag seines Vorgängers als Dekan, eines Wirtschaftshistorikers aus Harvard, 1927 einen kanadischen Wirtschaftshistoriker, Norman S. B. Gras, rekrutierte, der sich auf seinem Gebiet stark langweilte und so zum ersten Professor für Unternehmensgeschichte in Harvard und der Welt werden sollte. ${ }^{6}$ 
10 Dieser Lehrstuhl wurde von drei Führungskräften der großen Macy's-Kaufhauskette dotiert. Gras amtierte in den neuen Räumlichkeiten, deren Bau Donham auf der anderen Seite des Charles-Flusses zu Stande gebracht hatte. Selbst wenn die wissenschaftliche Zeitschrift, die Donham sofort ins Leben rief, wegen Meinungsverschiedenheiten zwischen Gras und dem Chefredakteur rasch wieder verschwand, war Gras in allen übrigen Belangen erfolgreich. Er unterrichtete die Studenten mit Hilfe der an der HBS hoch angesehenen Fallmethode, rekrutierte weitere Lehrkräfte, darunter eine Frau, und sammelte historisches Material $\mathrm{zu}$ den Entscheidungen und Transaktionen, auf dessen Grundlage er Artikel erarbeitete. Diese Lehrveranstaltungen und Artikel waren eine Art Gegenmittel, unter anderen, gegen die von der Krise von 1929 erneuerten Kritiken an Großunternehmen, deren Leiter $1934 \mathrm{zu}$ "Raubrittern" umgetauft wurden. 1939 ging er diesen Weg konsequent zu Ende: Er veröffentlichte das erste Lehrbuch der Unternehmensgeschichte, Business and Capitalism: An Introduction to Business History. Seine Problemstellung war eng definiert: $\mathrm{Er}$ behandelte nie direkt die Wirtschaftsgeschichte der Geschäfte, sondern konzentrierte sich auf die Verwaltung der Unternehmen und die Entscheidungsfindung der Angehörigen der Geschäftswelt. Trotzdem hat dieses Lehrbuch zwei dauerhafte Verdienste. Gras präsentierte den Kapitalismus als ein für das Verständnis der Unternehmensgeschichte unerlässliches System und als eine Kultur und er erarbeitete eine Klassifikation von fünf unterschiedlichen Typen von privatem Kapitalismus: den Kleinkapitalismus, den merkantilistischen Kapitalismus, den Industriekapitalismus, den Finanzkapitalismus und den Nationalkapitalismus. ${ }^{7}$

\section{Trotz Marc Bloch: Frankreich vollzieht die Wende nicht mit}

11 Ende 1929 wurde Gras von denjenigen, die gerade eine neue, ambitionierte französische Zeitschrift gegründet hatten, die Annales d'histoire économique et sociale (die erstmals am 15. Januar 1929 erschienen war), eingeladen, die neue Disziplin dort zu präsentieren. Diese Initiative Marc Blochs war im Grunde die Folge eines ganz anderen Projekts. Wie jüngst gezeigt wurde, hatten Marc Bloch und Lucien Febvre von 1919 bis 1926 über verschiedene Kontakte versucht, mit der moralischen und finanziellen Unterstützung amerikanischer Historiker der American Historical Association und der Rockefeller Stiftung eine europäische Zeitschrift zu gründen, die »absolut international, weltumspannend und kosmopolitisch" sein und den Namen Revue internationale d'histoire économique tragen sollte. Erst nach dem Scheitern dieses Projektes hatten sie eine rein französische Zeitschrift gegründet. ${ }^{8}$ Diese Initiative ist umso erwähnenswerter, als die Gründer der Annales damals in den USA nur sehr wenige Freunde hatten. In seinem kurzen, ganz zu Anfang des Jahres 1931 publizierten Artikel schrieb Gras, dass der besondere Blickwinkel der "Geschichte des Geschäftslebens«, wenn sie die »Organisation der Unternehmen und die Politik der Staaten« untersucht, der »Profit«, sei, »der durch den Einsatz von Arbeit und Kapital« erzielt wird. Die Orientierung, die er zum Ausdruck brachte, entsprach der Verankerung der neuen Disziplin hinsichtlich des Verständnisses des Kapitalismus. Indem er den Akzent auf das Studium der Praktiken und Entscheidungen der Akteure legte, beschloss er den Artikel mit einer emphatischen Ermahnung, die seine Orientierungen (ebenso wie seine europäischen Lektüren) enthüllte: »Weniger Marx und mehr Hegel.» $\aleph^{9}$ Hierbei ist 
anzumerken, dass der Übersetzer und die Redaktion sich damals dafür entschieden, business history mit »Geschichte der Geschäftswelt (histoire des affaires) « zu übersetzen. ${ }^{10}$

Der Artikel von Gras wurde durch einen bemerkenswerten Kurzbeitrag des KoDirektors der Annales, Marc Bloch ${ }^{11}$ eingeleitet, der 1995 in einer posthumen Aufsatzsammlung von seinem Sohn erneut publiziert wurde. ${ }^{12}$ Er wurde von einem der Biographen Blochs, der amerikanischen Historikerin Carole Fink, in ihrer Biographie von 1991 angemessen gewürdigt, danach 1995 von einem Unternehmensberater, der sich darauf spezialisiert hatte, auf Bestellung Unternehmensgeschichten zu schreiben. Schließlich wurde er, mit einer etwas anderen Perspektive, erstmals von Olivier Dumoulin in seiner geschichtswissenschaftlichen Habilitationsschrift kommentiert. ${ }^{13}$ Halten wir zunächst fest, dass Marc Bloch zu Beginn seines Artikels vor den USA den Hut zog und sich explizit von dem Misstrauen gegenüber diesem Land und seinem Kapitalismus abgrenzte, wie es in dem damals neu erschienenen vielgelesenen Reisebericht des Romanautors Georges Duhamels zum Ausdruck kam. ${ }^{14}$ Es ist interessant, die Argumentation Marc Blochs zur Grundlage der neuen Disziplin und zu ihren möglichen Anwendungsformen zu verfolgen.

Bezüglich der Grundlage wirft Marc Bloch die Frage nach den Beziehungen zwischen der neuen Disziplin und dem wirtschaftlichen Handeln auf. Er erfasst wunderbar die Bedeutung dieser Herausforderung:

»Einigermaßen detailliert über den Nutzen einer allgemeinen Kenntnis der Wirtschaftsgeschichte für den Geschäftsmann zu diskutieren, das würde wohl nahezu darauf hinauslaufen, das gesamte Problem der Geisteswissenschaften aufzuwerfen."

Eine bemerkenswerte Beobachtung.

14 Marc Bloch unterstützte die neue Disziplin vorbehaltlos - und damit auch die Notwendigkeit, Unternehmensarchive zu entwickeln. Aber was ihre Namensgebung betraf, zuckte er zurück: Er sprach von »diesem seltsamen Begriff der >Geschichte der Geschäftswelt`(l'histoire des affaires [business history])«. Wir werden noch sehen, dass diese Debatte Folgen haben würde.

Im Gegensatz dazu ging er in Bezug auf die Idee, dass diese neue Geschichte "ganz von der Idee des Präzedenzfalles, fast in einem juristischen Sinn des Wortes dominiert wird, die, wie man weiß, den angelsächsischen Rechtssystemen besonders vertraut ist« $\mathrm{zu}$ Gras auf Distanz. Er verglich mit Vehemenz »diese Art, die Verwendung historischen Materials zu konzeptualisieren oder zu präsentieren« mit dem Gebrauch, den die »Kriegskunst" davon macht, und er unterstrich die "schweren Unannehmlichkeiten, die dadurch entstanden sind«. Er hielt ihm eine nach wie vor aktuelle Geschichtsauffassung entgegen:

"Ich bezweifle nicht, dass das Verständnis der Phänomene der Gegenwart seinen Nutzen aus dem Studium fast gleichartiger Phänomene ziehen muss, die die Vergangenheit anbietet. Aber unter einer Bedingung: dass man den Akzent sorgfältig auf das `fast` legt, oder, mit einem Wort, auf die Unterschiede ebenso wie auf die Ähnlichkeiten. Die Geschichte ist vor allem eine Erkenntnis der Wandlungsvorgänge. Vielleicht ist es gerade diese Eigenschaft, die für die Praxis am nützlichsten ist.«

16 In seinem Buch von 2003 kommentierte Olivier Dumoulin diese Geschichtsauffassung zu Recht mit folgenden Worten: "[D]ie Verwendung vergangener Ereignisse $\mathrm{zu}$ Vergleichszwecken, zum Messen der Unterschiede, macht aus der Geschichte eine 
Experimentalwissenschaft, die dazu geeignet ist, den Geist zu einer Analyse des Unbekannten anzuregen. « $^{15}$

Marc Bloch zog die Konsequenzen aus seinen Überlegungen und wandte sich den Beziehungen zwischen Geschichte und Betriebswissenschaft zu. (Dieser Ausdruck stammt natürlich nicht aus der damaligen Zeit) - zunächst für die Ausbildung der Manager. Er begrüßte die Initiativen von Donham, auch wenn er ihn nicht ausdrücklich nannte, und er verwandte Amerika, um Frankreich zu kritisieren. Er tat es hinsichtlich der Grundausbildung und bereits mit einem Plädoyer für die longue durée:

»Die Handelshochschule von Boston unterrichtet Geschichte: eine frappierende Lehre für Einrichtungen vom gleichen Typ, die bei uns, wenn ich mich nicht irre, in den meisten Fällen jedes historische Studium ganz bei Seite lassen. Sie aber lässt diese Geschichte im genuesischen Mittelalter beginnen."

Bloch hielt sein Plädoyer auch für die Fortsetzung der Ausbildung, die er dem "Niedergang durch eingefahrene Routinen « gegenüberstellte. Dieser Mediävist, der so sensibel für das war, was er "die aktuellste Gegenwart", nannte, nahm auf die Schaffung des "Vorbereitungszentrums für Wirtschaft und Handel« (Centre de Préparation aux Affaires) Bezug, das im Vorjahr, 1930 von der Pariser Handelskammer gegründet worden war. ${ }^{16} \mathrm{Er}$ beurteilte es sehr positiv: »Der Versuch ist glücklich, der Studienplan sehr intelligent entworfen.« Trotzdem zog er sofort den Vergleich mit Harvard, das dem CPA als Modell gedient hatte - einschließlich der Einführung der Fallmethode in Frankreich:

"Die neue Institution hat zwar die Graduate School of Business Administration von Boston ausdrücklich als Vorbild gewählt, mit der sie einen echten Informationsaustausch organisiert hat, aber zumindest in einem Punkt hat sie es abgelehnt, ihrem Modell zu folgen: In Paris gibt es keinerlei historische Lehrveranstaltungen."

Marc Bloch sprach auch an, wie die Beziehung zur Geschichte der Führungskräfte und der öffentlichen Entscheidungsträger aussehen könnte. Was die Grundlage betrifft, so plädierte er nicht nur für die Unternehmensgeschichte: Er argumentierte, dass die Geschichte im Allgemeinen "für den praktisch orientierten Menschen eine wunderbare, unerlässliche Schule psychologischer und sozialer Analyse« sei. Er fügte eine weitere, innovative Dimension hinzu: »Die Lehrmeinung oder die Mythen sind einfach und klar. Aber die menschlichen Elemente und die mehr oder weniger unbewussten tiefen Bedürfnisse sind es weitaus weniger."

20 Und er kam zu dem Schluss: „Und wie soll man diesen menschlichen Hang zur Analyse von Massen befriedigen, der für das Handeln so notwendig ist, wenn nicht mit Hilfe der Geschichte, sofern sie angemessen verstanden und unterrichtet wird?«

21 Er befragte die Entscheidungsträger (und die Wissenschaftler) zur Gegenwart: »Für sich betrachtet, kann sie sich höchstens beschreiben, aber nicht verstehen.« Er gab zwei Beispiele. Das eine stammte aus der Aktualität der Welt der Nachkriegszeit:

»Wenn heute so viele Staatsmänner und Finanziers den Währungskrisen hilflos gegenüberstehen, dann deshalb, weil [...] sie daran gewöhnt sind, in der Gegenwart zu leben und nur an sie zu denken. So hatten sie sich daran gewöhnt, die Stabilität der Instrumente des Austauschs für ewig zu halten. Ein zutreffenderer Sinn für die Geschichte hätte sie dazu veranlasst, sie als ein Phänomen jüngeren Datums und von unbestimmter Dauer anzusehen, als eine der Übergangserscheinungen eines ewigen Werdens." 
andere Beispiel bezog sich auf die Führungskräfte der Unternehmen. Marc Bloch warnte sie vor »diesen gedächtnislosen Geschöpfen, die Großunternehmen allzu oft sind « und vor der »zum großen Teil illusionären« Vorstellung, die für »eine Bank, eine Fabrik, ein Handelshaus« darin bestehe, zu glauben, dass sie »ausschließlich von der Gegenwart abhängen, oder, wenn man so will, von einer unmittelbaren Vergangenheit, die sich schnell überlebt hat «. Indem er als Historiker die Ideen von Donham und Gras unterstützte, begnügte er sich nicht damit, in diesem Sinne zur Erhaltung und Sichtung der Unternehmensarchive aufzurufen (er hatte bereits am 20. September 1929 an Lucien Febvre geschrieben, es sei dringend, eine Untersuchung zu den Archiven von Privatunternehmen anzuregen. Dies geschah dann 1930 durch eine Notiz eines Generalinspektors der Bibliotheken und Archive in den Annales, die durch einen Vorspann von Lucien Febvre eingeleitet wurde, in der er an die diesbezüglichen Aktivitäten der Business Historical Society Donhams und Gras' erinnerte ${ }^{17}$ ). Er sähe schon »in den Studienbüros unserer bedeutendsten Häuser neben dem Leiter des Statistikabteilung - übrigens: existiert denn eine solche Abteilung immer noch? - einen darauf spezialisierten Historiker« sitzen. Zu Beginn seines Artikels stützte er sich auf Informationen, die zuvor in den Annales ${ }^{18}$ erschienen waren, um mit Nachdruck auf die Bewegung hinzuweisen, die »in diesen USA, die die Avant-Garde des praktischen Lebens von heute sind [...], die modernsten Geschäftsleute der Welt dazu veranlasst, sich für die Wirtschaftsformen der Vergangenheit zu interessieren«.

Trotz der Publikation des Aufsatzes von Gras und des Aufrufes Marc Blochs, in Frankreich die Unternehmensgeschichte weiterzuentwickeln, befolgte ihn bis zum Zweiten Weltkrieg der größte Teil der französischen Historiker nicht - abgesehen davon, dass sie, im Vergleich zum "amerikanischen Vorbild" "deutliche Nuancen" eingeführt hatten. Dies gilt zumindest, wenn ich mich auf die Auswertung der Annales von 1929 bis 1940 beziehe, die ich für diesen Beitrag durchgeführt habe. Das lag nicht am Mangel an Beharrungsvermögen von Seiten der Annales-Direktoren. Der KoDirektor Lucien Febvre machte sich bezüglich der Unternehmensarchive zwei Nummern nach dem Artikel von Marc Bloch erneut ans Werk, indem er das "amerikanische Vorbild« zitierte. ${ }^{19} 1935$ veröffentlichten die Annales einen Aufruf zur Erhaltung und Benutzung von Unternehmensarchiven in Großbritannien..$^{20}$ Aber wenn auch das Rechnungswesen oder die Kommanditgesellschaft Gegenstand von Artikeln waren, so findet man in elf Jahren doch nur einen Artikel und eine historische Miszelle zur Unternehmensgeschichte. Beides war zudem noch das Werk derselben Person. ${ }^{21}$ Woran lag das? Anhand des Inhalts der Annales können drei Antworten vorgeschlagen werden: das massive Interesse der französischen Wirtschaftshistoriker dieser Zeit für die Wirtschaftskrisen, die Währung und die Preisgeschichte; der Mangel an zugänglichen Unternehmensarchiven, die, im Gegensatz zu diesen Verhältnissen, der Nährboden der neuen Disziplin in Amerika gewesen waren; das fehlende Interesse der französischen Handelshochschulen (écoles de commerce), das Marc Bloch angeprangert hatte. Aber die Unternehmen waren in den Annales durch die Feder von Wirtschaftswissenschaftlern, Geographen und Soziologen vertreten. So publizierte 1935 der Soziologe Georges Friedmann einen Artikel über die tschechische Schuhfirma Bat'a. 22

In der Nachkriegszeit schlug die neue Disziplin Wurzeln und konfigurierte sich neu. 


\section{Universalisierung und Differenzierung}

Nach dem Zweiten Weltkrieg dehnte sich die Unternehmensgeschichte nach und nach auf die gesamte industrialisierte Welt aus, nahm dabei jedoch unterschiedliche nationale Ausprägungen an. ${ }^{23}$ In Kontinentaleuropa zog sie sogar einen Teil der Marxisten an. Aber die Entwicklung verlief nicht geradlinig. Auch wenn sie in den USA im Kontakt mit der Soziologie schließlich einen attraktiven wissenschaftlichen Ansatz fand, so geschah das doch um den Preis zweier wichtiger Infragestellungen.

\subsection{Die USA}

Nach dem Krieg machte die von Gras angestoßene Problematik wegen fehlender neuer Verallgemeinerungen kaum noch Fortschritte und sie wurde auf zwei entgegengesetzte Arten in Frage gestellt.

Eine davon war die Firmengeschichte (company history). Es handelte sich um sehr detaillierte Monographien, in denen man aber nicht immer Gesamtschauen oder allgemeine Perspektiven vorfand.

Die andere war die Unternehmer-Geschichte (entrepreneurial history), die 1948 in Harvard selbst als Reaktion auf die business history und ihre relative Stagnation entstand. Historiker dieser Universität von der anderen Seite des Charles-Flusses gründeten ein Research Center for Entrepreneurial History, das den Wirtschafswissenschaftler Joseph Schumpeter zu seinen wichtigsten Mitgliedern zählte und mehrere junge Historiker, sowohl Ausländer als auch Amerikaner. Die Kontakte zu dem Soziologen Talcott Parsons führten bei diesen jungen Historikern ein Zusammentreffen der Dynamik Schumpeters mit den sozialen Kategorien Max Webers herbei. Aber Schumpeter starb 1953, die Rockefeller-Stiftung erneuerte ihre Subventionen nicht und das Zentrum schloss 1958 seine Tore, nachdem es sich einerseits auf den Unternehmergeist (entrepreneurship) und andererseits auf das Ausland konzentriert hatte. Der Kapitalismus genoss dort allerdings keinen Vorrang. ${ }^{24}$ Eines der bedeutendsten Mitglieder des Zentrums und einer der wichtigsten Autoren dieser Zeit, Thomas C. Cochran, stellte die Position des Unternehmens in der Gesellschaft in den Mittelpunkt. Er fügte den Arbeiten seiner Kollegen ein PionierInteresse für Gruppen-Normen und Geschäftsmentalitäten hinzu, eher für Kultur als für Institutionen, kurz gesagt, eine Kulturgeschichte der Unternehmen. ${ }^{25}$

Ein Ausweg aus diesen beiden Infragestellungen wurde von Alfred Chandler in Strategy and Structure. Chapters in the History of the American Industrial Enterprise (1962) gefunden. Unter dem dreifachen Einfluss seiner Kriegsjahre, seiner Kontakte mit dem Soziologen Talcott Parsons und seiner Jahre am Research Center for Entrepreneurial History schlug er das Konzept der Strategie vor, das er wechselseitig mit den Unternehmensstrukturen verknüpfte. Er wertete die leitenden Angestellten auf und bot einen synthetischen chronologischen Rahmen zum Verständnis der Entwicklung der amerikanischen (Groß-)unternehmen seit der Mitte des 19.Jahrhunderts an. Seine Überzeugungen dominierten bis in die Mitte der 1990er Jahre die business history, die sich zu einer echten Geschichte der Unternehmen entwickelte - und $\mathrm{zu}$ einer neuen Art, Wirtschaftsgeschichte zu betreiben. 

die ihrerseits das Modell Chandlers obsolet werden ließen. Einerseits brachten Philip Scranton und andererseits Charles F. Sabel und Jonathan Zeitlin die Bedeutung von Netzwerken kleiner und mittlerer Unternehmen in die Debatte ein - und die der flexiblen Spezialisierung als Alternative zur Massenproduktion, die mit ihr koexistierte. ${ }^{26}$ Auf der anderen Seite lehnten Naomi Lamoreaux, Daniel Raff und Peter Temin die Einfachheit der Dichotomie zwischen den Märkten und den Hierarchien von Unternehmen ab und legten den Akzent auf die strukturierenden Effekte der Asymmetrie der Information, die Vielfalt der Koordinationsmechanismen der Wirtschaft zwischen den Märkten, die individuelle Transaktionen erleichtern, und auf die Hierarchien, die dauerhafte Strukturen für die wiederholten Transaktionen liefern. Sie unterstrichen den vor kurzer Zeit erfolgten Niedergang der Bedeutung der großen, hierarchischen Unternehmen, die nicht die einzig mögliche Entwicklung für die jeweilige Form des Kapitalismus darstellen. ${ }^{27}$

\subsection{Großbritannien}

Die Briten zeigten gleichermaßen ihr Interesse an dem, was sich in den USA abspielte, und ihre Bemühung darum, sich davon zu unterscheiden, und dies umso mehr, als sie bis 1962 keine business schools haben wollten. ${ }^{28}$ Erst 1934 gründete eine Gruppe von Universitätsangehörigen und Unternehmenschefs den Business Archives Record Council, der die Erhaltung und die Katalogisierung von Unternehmensarchiven propagiert und jährliche Tagungen organisiert. Wir haben weiter oben bereits gesehen, dass diese Initiative 1935 in den Annales Widerhall fand. Von diesem Anstoß aus entwickelte sich die äußert aktive Unternehmensgeschichte der Nachkriegszeit, die sich entweder für die Gründung und Weiterentwicklung der Familienunternehmen seit dem 18. Jahrhundert oder für die Gründe des Wachstums der Großunternehmen und die Entwicklung ihrer internen Strukturen interessierte. 1958 wurde eine Zeitschrift namens Business History gegründet. ${ }^{29}$ Während der Marxismus einen sehr dynamischen Teil der Sozial- und Wirtschaftshistoriker anzog, hatte er bei Unternehmenshistorikern keinen großen Erfolg.

\subsection{Zwei Besiegte des Zweiten Weltkrieges}

Sowohl in Japan als auch in Deutschland wurde ein Teil der Kriegsschuld den Unternehmen zugeschrieben und diese Anschuldigungen trugen zur Entstehung einer nationalen Unternehmensgeschichte bei.

In Japan entstand die Unternehmensgeschichte als Reaktion auf die zeitweilige Vorherrschaft des Marxismus in den wirtschaftswissenschaftlichen und, in einem weit geringeren Umfang, in den geschichtswissenschaftlichen Fakultäten der Universitäten. Die Unternehmensgeschichte bietet seitdem eine andere Geschichte an: eher die der dominierenden Akteure, als die der Strukturen bzw. die einer liberal-staatlichen Sicht der Wirtschaft und einer Weltsicht, die sich von der Westlichen unterscheidet. Sie fand die Unterstützung eines Teils der Führungskräfte der Unternehmen. Auf diese Weise entwickelte sich eine Geschichte, die danach strebte, die Originalität und die nationale Größe zu verstehen, die aber nicht über einen gefestigten Theorierahmen verfügte. Sie brachte deshalb Arbeiten hervor, von denen einige sehr originell waren und bereitete 
gleichzeitig, dank Keiichiro Nakagawa, einem ehemaligen Mitglied des Research Center for Entrepreneurial History, der Problematik Chandlers einen ausgezeichneten Empfang.

In der Bundesrepublik Deutschland lässt sich für monographische Arbeiten ebenfalls die Unterstützung durch eine erhebliche Anzahl von Unternehmen aus dem Bereich der Banken, der Industrie und des Handels feststellen - und die Unternehmensgeschichte grenzte sich gegenüber der Wirtschaftsgeschichte $a b$, indem sie sich 1956 eine Zeitschrift mit dem besonders symbolträchtigen Titel »Tradition« zulegte. Mit Ausnahme von Fritz Redlich, der in die USA emigriert und dann an den Arbeiten des Research Center for Entrepreneurial History beteiligt gewesen war, positionierte sie sich als Gegenpol zur (allgemeinen) westdeutschen Historiographie, die insbesondere von dem Gespenst der NS-Zeit und in einem weiteren Sinn von der Kritik am Sonderweg umgetrieben wurde, von einer wirtschaftlichen Modernisierung, die nicht von einer entsprechenden politischen Demokratisierung begleitet gewesen sei. Sie privilegierte stattdessen den Dialog mit den Unternehmern. Die intellektuelle Erneuerung und der wissenschaftliche Charakter dieser Geschichte hatten mehrere Quellen: der deutschen Sozialgeschichte, dem amerikanischen Einfluss und schließlich, in den 1980er Jahren, aus einer kritischen, theorieverliebten Unternehmensgeschichte. Der Titel ihres Bulletins brachte ihre Ausrichtung gut zum Ausdruck: »Akkumulation«. ${ }^{30}$

\subsection{Zwei romanische Länder}

Frankreich erlebte eine frühzeitigere Entwicklung und setzte auf eine andere Karte. Hier verbanden sich in der Tat marxistische und nicht-marxistische Historiker. Auf Seiten der zuerst genannten finden wir Jean Bouvier, zunächst ein Bankhistoriker. Auf der Seite der Zweiten Bertrand Gille, Claude Fohlen und Pierre Léon, Bank-, Industrieund Handelshistoriker. Die einen wie die anderen unterschieden sich von den Wirtschaftshistorikern - die, als Echo auf die Wirtschaftswissenschaften mit Keynes'scher Dominante (das Kapital, das Gehalt, der Profit, die Rendite), makrohistorisch dachten - durch die Wahl einer mikrohistorischen Perspektive und durch eine sehr starke Wiederaufwertung der Akteure im Vergleich zum dominierenden Trio Strukturen - Konjunkturen - Preise. Diese neue Disziplin nahm nicht die 1931 von den Annales gewählte Terminologie von der "Geschichte des Geschäftslebens« (»histoire des affaires«) auf, die dennoch von Fernand Braudel als Titel einer der Publikationsreihen beibehalten wurde, die die neue sechste Sektion der École des hautes études veröffentlichte: "Affaires et gens d'affaires" (Geschäfte und

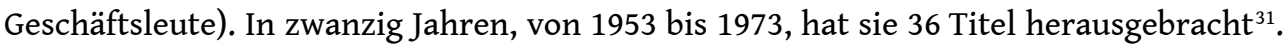
Andere Reihen, zum Beispiel "Industrie et artisanat" (Industrie und Handwerk), nahmen ebenfalls die neue Disziplin auf. Der Kapitalismus war ein beiden Strömungen der Unternehmensgeschichte gemeinsamer Faktor. So erschienen auf der nichtmarxistischen Seite in der Reihe »Affaires et gens d'affaires« 1959 unter dem Titel Recherches sur la formation de la grande entreprise capitaliste (1815-1848) [Forschungen zur Entstehung des kapitalistischen Großunternehmens (1815-1848)] die thèse complémentaire von Bertrand Gille und auch das erste Buch von Guy Thuillier, das gleichermaßen von Paul Leuilliot und Maurice Lévy-Leboyer inspiriert wurde: Georges Dufaud et les débuts du grand capitalisme dans la métallurgie en Nivernais au XIX siècle. [Georges Dufaud und die Anfänge des Großkapitalismus in der Metallurgie des Nivernais im 19. Jahrhundert]. Andererseits veröffentlichte die École 1965 das, was bis heute die einzige historische Gesamtuntersuchung zum kapitalistischen Profit im 
19. Jahrhundert auf der Ebene einer Nation auf der Grundlage von Forschungen zu drei Sektoren geblieben ist: zu Kohlebergbau, Eisen- und Stahlindustrie und Banken. ${ }^{32}$

In derselben École, aber in einer anderen Sektion, gründete der Historiker Bertrand Gille 1953 »mit fast nicht existierenden Mitteln« eine Forschungsgruppe zur Geschichte von Unternehmen, die zunächst vier Ausgaben eines mit Matrize vervielfältigten

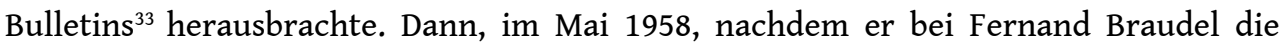
Aufnahme in seine Gruppe am Centre de Recherches Historiques der sechsten Sektion der École ${ }^{34}$ erreicht und "umfangreichere Mittel« erhalten hatte, entwickelte er vier Forschungsachsen, darunter »die Struktur des Kapitalismus in Frankreich" (»la structure du capitalisme en France«). Gille gründete eine halbjährlich erscheinende Zeitschrift, die er auf den Namen Histoire des entreprises (Unternehmensgeschichte) taufte. Dieser Terminus sollte sich schließlich in Frankreich durchsetzen. Gille schrieb in der ersten Ausgabe:

"Diese neue Disziplin hat in den USA und in einigen europäischen Ländern eine große Verbreitung erreicht. [...] Wir hoffen, dass sie [unsere Zeitschrift] dieser Kette ein weiteres Glied hinzufügt und dass sie dazu dienen wird, unerlässliche Verbindungen herzustellen. « $^{35}$

Dieses Programm war ein Echo auf den Wunsch des stark von der Rockefeller-Stiftung unterstützen Zentrums, »ähnliche Wege auszuprobieren, wie die der amerikanischen Unternehmensgeschichte ${ }^{36}$

Dennoch wurde die Zeitschrift bereits nach zwölf Ausgaben und, offiziell, nach sechs Jahren eingestellt. Die letzte Ausgabe mit Datum vom November 1963 erschien jedoch erst 1965. Mit Bezug auf die "Harvard-Historiker, die die Initiatoren dieser Bewegung gewesen waren«, schrieb Bertrand Gille:

"Das Thema ist sicherlich nicht erschöpft. Aber es schien uns so, als ob die Unternehmensgeschichte inzwischen ihr Bürgerrecht in der französischen Geschichtsforschung erhalten hat. 1 $^{37}$

Das »Bürgerrecht" gab es sehr wohl. Aber dieses Verschwinden, über dessen Gründe man diskutierten kann (insbesondere hatte die Zeitschrift kein Redaktionskomitee und sie publizierte nur Arbeiten eines Teils der auf diesem Gebiet aktiven Historiker), trug dazu bei, dass es »in Frankreich nur zu einer unvollständigen Institutionalisierung der Unternehmensgeschichte«, kam, sei es wegen der »Heterogenität der theoretischen Bezugnahmen zwischen dem Milieu der französischen Kultur und dem amerikanischen Milieu« oder wegen der fehlenden Umsetzung »dieser Integrationsstrategie zwischen Wirtschaftspraxis und Wirtschaftsgeschichte.$^{38}$

Italien wandte sich erst zu Beginn der 1970er Jahre der Unternehmensgeschichte zu. ${ }^{39}$ Bis dahin hatten die Unternehmen kein Vertrauen zur Geschichtswissenschaft und interessierten sich nicht für Archive. Was die Wissenschaftler anbelangt, so trafen sich die idealistischen und die marxistischen Orientierungen in diesem Punkt und veranlassten viele dazu, die Unternehmensgeschichte nicht als einen sinnvollen Gegenstand zu betrachten. Drei Pionieren gelang es, die Blockade zu brechen, und die Unternehmensgeschichte erlebte ab den 1980er Jahren ihre volle Entfaltung. Diese späte Entwicklung war zu einem guten Teil den Anstrengungen von Historikern zu verdanken, die aus der Studentenbewegung hervorgegangen waren oder die sich an radikalen oder marxistischen Gruppen beteiligt hatten. Sie wollten sich an der Erforschung der Rolle von Unternehmen unterschiedlicher Größe für 
Wachstumsprozesse, innerhalb der Gesellschaft und in ihrer Beziehung zum italienischen Staat, beteiligen.

\section{Erkenntnisse der Unternehmensgeschichte zum französischen Kapitalismus}

Die Präsentation, die wir gerade von der allmählichen und ungleichmäßigen Entwicklung der Unternehmensgeschichte seit 1945 und ihrer Beziehung zum Kapitalismus und von ihrer je nach Land unterschiedlichen Ausdifferenzierung gegeben haben, hat eine große Zahl von Themen und möglichen Methoden zu Tage gefördert, die auch in Bezug auf Frankreich aufgenommen worden sind. Das erlaubt es uns nun, den Platz der französischen Unternehmen im Kapitalismus und bestimmte spezifische Aspekte des französischen Kapitalismus anzusprechen.

\subsection{Ein doppelter Kapitalismus}

41 Die Studien haben auf diese Weise für Frankreich wie für alle anderen Länder die Bedeutung eines Kapitalismus von Familienunternehmen gezeigt. Dies gilt zunächst natürlich für die Kategorie der kleinen und mittleren Unternehmen, einschließlich ihrer Funktion für die wirtschaftliche Integration von Mitgliedern eingewanderter Bevölkerungsgruppen, danach aber auch, besonders im Bereich des Vertriebs, für den Sektor der Großunternehmen. Man hat hier eine echte Kehrtwende der Historiographie erlebt. Ursprünglich hatte man den Familien-Kapitalismus als ein Element der Schwäche angesehen, weil viele Familienunternehmen Emotionen ausgesetzt sind, die die Familien erschüttern können oder weil sie nicht lange überleben. Oder man hatte sie sogar als Element der »Rückständigkeit« eingestuft, weil ihre Führungskräfte die Profite nicht in die Wirtschaft, sondern in Grundbesitz, Immobilien oder die Kunst investieren würden. Heute erblickt die Historiographie in diesem Teilbereich des Kapitalismus nicht nur ein Element der Kreativität und der kontinuierlichen Erneuerung der Wirtschaft, sondern, indem sie gleichzeitig seine Strategien und Vererbungsformen untersucht, einen Willen zur Dauerhaftigkeit und damit eine Ablehnung der Kurzfristigkeit, die nunmehr den am stärksten mit den Finanzinstitutionen und -märkten verbundenen Unternehmen zugeschrieben wird. ${ }^{40}$

Indem sie dieses erste Argument verallgemeinert und theoretisiert hat, zeigte Claire Lemercier, dass der erste französische Kapitalismus bis in die 1880er Jahre vom Kleinund Großhandel getragen wurde, der die Nachfrage in kleinteiligen, Kaufleuten untergeordneten Netzwerken auslöste bzw. sie befriedigte, um sehr differenzierte Waren zu produzieren. In ihren Augen ist "das, was sich im Vergleich zu den ersten, von Fernand Braudel beschriebenen Kapitalisten verändert hat, vor allem eine Frage des Maßstabs: Der Fernhandel hat enorm zugenommen, seine Produkte sind auf andere Märkte geströmt, aber seine unternehmerischen Gepflogenheiten, sein Recht und seine Organisationsformen haben sich lange Zeit ohne radikale Veränderung bewahrt«. ${ }^{41}$

Gleichzeitig entwickelte sich ein anderer Kapitalismus, der des Großunternehmens, der lange Zeit in der Minderheit blieb: in der Metallverarbeitung, dem Bergbau, der Glasindustrie und der Chemie. Aber er setzte sich bei den Eisenbahnen, dem Gas oder im Dienstleistungssektor mit den Kaufhäusern oder den Depot-Großbanken durch. Einige von diesen Unternehmen wurden multinational, wie zum Beispiel 1858 Saint- 
Gobain in Deutschland oder die Pathé-Kinos, Weltmarktführer in den 1900er Jahren. Das 20. Jahrhundert war Zeuge der Versuche dieser Großunternehmen, die Übrigen zu koordinieren oder zu dominieren und die des Staates, der lange Zeit noch nicht über angemessene Möglichkeiten dazu verfügte, die privaten oder öffentlichen Großunternehmen immer mehr zu unterstützen. ${ }^{42}$

\subsection{Konkurrenz} hat sich, von den Unternehmen ausgehend, im Hinblick auf die Konkurrenz vollzogen. Da er auf die Gesetzgebung und die Verordnungen zurückging, die aus der Französischen Revolution hervorgegangen waren, die die Korporationen abgeschafft hatten, war der französische Kapitalismus von den Historikern und Soziologen lange als strukturell verschieden vom deutschen Kapitalismus angesehen worden, der als kooperativ präsentiert wurde. Die seit den 1970er Jahren begonnenen Forschungen haben die Kluft zwischen der gesetzlichen Norm und der Wirklichkeit gezeigt. Auf der einen Seite versuchten zahlreiche lokale Organisationen, die kollektiven Regeln in Kraft zu setzen und sowohl Aktivitäten als auch Ausgabenquellen aufzuteilen. ${ }^{43}$ Andererseits wurde der 1810 erlassene Artikel 419 des französischen Strafgesetzbuchs, der das Werk der Revolution fortsetzte, indem er Absprachen und Kartelle verbot, von den Gerichten wenig angewandt, bevor er schließlich 1926 abgeschafft wurde. So kann man annehmen, dass der französische Kapitalismus aus der Kooperation viel Energie bezieht, zwar sicherlich weniger als sein deutscher Nachbar, aber dennoch nicht so weit davon entfernt. ${ }^{44}$ Schließlich implizierte die Entwicklung der öffentlichen Monopole und der öffentlichen Unternehmen noch eine andere Beschränkung der Konkurrenz. So wurden nach dem politischen Umschwung von 1986 Konkurrenzpraktiken von den staatlichen französischen Instanzen (und der Europäischen Kommission in Brüssel) systematisch gefördert. Aber seitdem sanktionieren die Autoritäten jedes Jahr wie bei den Köpfen der Hydra von Lerna das Wiederaufkommen illegaler Absprachen.

\subsection{Landwirtschaft}

Während die Landwirtschaft bestens dafür bekannt ist, dass sie eine der Ursprungsquellen des englischen Kapitalismus war, haben neuere Forschungen gezeigt, dass das in Frankreich in dieser Form nicht der Fall war. Der Eintritt der Landwirtschaft in den Kapitalismus erfolgte allmählich und auf unterschiedlichen Wegen. ${ }^{45}$ Obwohl es im Pariser Becken seit dem 17.Jahrhundert Großbauern gab, die über ein umfangreiches Personal verfügten, so war es doch im 19. Jahrhundert die Einführung von Maschinen zur Reduzierung der Personalkosten, die in der Betriebsführung den wachsenden Rückgriff auf Kapital auslöste. Zu den Folgen gehörten die Steigerung der Produktivität, die Vergrößerung technischer und landwirtschaftlicher Kompetenzen, die Ausweitung der Buchhaltungsüberlegungen, das Anwachsen der Verschuldung, und, insgesamt gesehen, das Aufkommen des landwirtschaftlichen Unternehmens, das lange Zeit vor allem ein Familienunternehmen war. Die kapitalistische Rationalisierung wurde staatlicherseits seit dem Ende des Zweiten Weltkriegs gefördert. Aber es kam erst vor Kurzem dazu, dass sich die (Waren-)Termingeschäfte, der Zustrom von Kapital aus Finanzen und die Grundbesitz-Konzentration entwickelten.

Trivium, 28 | 2018 


\subsection{Arbeit} war lange Zeit mit sehr alten Arbeitsweisen vereinbar: der Gruppenarbeit oder Akkordarbeit, bei der das Unternehmen nicht selbst die Arbeiter, die Landarbeiter oder - in einem Teil der Kolonien bis 1848 - die Sklaverei oder zwangsverpflichtete Arbeitskraft verwaltete. Die regionale Streuung der Gehälter blieb ein Jahrhundert lang stark ausgeprägt. Der Arbeiter als Gehaltsempfänger im Großunternehmen war also in der Minderheit im Verhältnis zu den Handwerkern oder zu den selbständigen oder halb-selbständigen Arbeitern, die per Werklohnvertrag von den Auftraggebern, von denen sie durch eine sozial sowohl von den Arbeitgebern als auch von den Arbeitern akzeptierten Norm abhingen, bezahlt wurden. Ebenso im Verhältnis zu Bauarbeitern und solchen, die bei öffentlichen Arbeiten auf den Baustellen tätig waren. Der Übergang von der Bezahlung eines vollendeten Werkes, die von Vornherein vereinbart war, zur Bezahlung der Arbeitszeit vollzog sich langsam. Der Status des Arbeitnehmers, bei dem durch einen Arbeitsvertrag die Unterordnungsmodalitäten, die Ungleichheit zwischen Frauen und Männern bei der Arbeit und, schließlich, die Haftungsbedingungen des Arbeitgebers präzisiert wurden, kam erst am Ende des 19. Jahrhunderts auf und wurde unter den Auswirkungen des Drucks der Arbeiterschaft, der Tätigkeit bestimmter Juristen und des Staates ${ }^{46}$ zunehmend zur Norm - besonders ausgeprägt im Sektor der Großunternehmen. ${ }^{47}$ Er erreichte auch das White-Collar-Milieu: Banken, in denen man seine Karriere ohne Diplom begann, ${ }^{48}$ Eisenbahnen und Geschäfte.

Diese Sichtweise der Rolle der Unternehmen in den Umbruchsprozessen des französischen Kapitalismus wirft zwei viel diskutierte Fragen auf: nach der Rolle der Banken und nach der Charakterisierung der modernen Unternehmen.

Die französischen Unternehmenshistoriker haben ihre Ansichten zu den Banken geändert. Sie analysieren sie zweifelsohne in ihrer ganzen Komplexität, als Sammler von Kapital, als Kreditgeber für Unternehmen, für Verwaltungen und seit kurzem für Konsumenten, als Investoren, als Vermittler in den Beziehungen zu Finanzmärkten, als Kontrollagenten, als Manager ihres Personals und als sensible Akteure in den Perioden wirtschaftlicher oder finanzieller Krisen. Wenn man die Bandbreite von Finanzierungsmethoden betrachtet, die anderen Unternehmen zur Verfügung stehen (von den Familien bis hin zu Notaren, der Selbstfinanzierung, der Finanzvermittlung und den Aktien- und Obligationsportfolios der Banken), dann scheint es so zu sein, dass eine ganze Reihe von französischen Banken und Börsen spontan kein kohärentes letztes Wort bei der Organisation von Märkten findet, so wie das bei dem deutschen Nachbarn der Fall ist, sondern dass sie jeweils ihr eigenes Spiel spielen, ein Verhalten, das unter bestimmten Umständen den Anteil langfristiger Aktionen bremsen kann, ohne die wirtschaftliches Handeln beschränkt bleibt.

Das Aufkommen moderner Unternehmen, vor allem seit dem Ende des 19. Jahrhunderts, war durch den Aufstieg professioneller Führungskräfte, den Rückgriff auf die Forschung zur Produkterneuerung und auf Dienstleistungen gekennzeichnet und durch eine spezifische Rolle des Unternehmens, das nicht nur eine rein finanzielle Einrichtung war, die Kapital akkumulierte und gewährte und ihre Profite verteilte, sondern ein Kollektiv von Innovation und Kampf, das sich organisierte, um bisher noch 
nicht umgesetzte Strategien $\mathrm{zu}$ verwirklichen, und das durch die öffentliche Regulierung eingerahmt werden konnte. Es sah sich einem wachsenden Einfluss der Konsumenten auf die Entwicklung, den Gebrauch und das Schicksal der Objekte ausgesetzt. ${ }^{49}$ Die Arbeit der Historiker und der Soziologen (siehe die Artikel von Hervé Joly und von Pierre François und Claire Lemercier in dieser Trivium-Ausgabe) zeigen, dass die dreifache Bewegung der Privatisierung öffentlicher Unternehmen, der Internationalisierung des Kapitals der Großunternehmen und der Finanzialisierung der Wirtschaft, die Frankreich ungefähr Ende der 1980er Jahre erreichte, die Praktiken und die Werte der Führungskräfte veränderte, aber weitaus weniger ihre Herkunft und ihre Netzwerke.

\section{Schlussbemerkungen}

Die Entwicklung der Unternehmensgeschichte hat auf diese Weise mehrere Folgen für das Verständnis der Geschichte des Kapitalismus. ${ }^{50}$ Sie unterstreicht die verschiedenen Maßstäbe, die sie sich gleichzeitig zu eigen machen kann: von der Mikrohistorie - dem Bauernhof, der Werkstatt, dem Büro, dem Laden, der Agentur, der Internetseite - zum Internationalen - dem Kreislauf von Produkten und Ideen, Wertschöpfungsketten, den Haupt-Finanzmärkten. Sie platziert die Unternehmen an den Vorposten zweier Hauptfragen, die ebenso zentral wie konfliktträchtig sind: die der geographischen Territorien und die der Globalisierung. Sie stellt die Frage nach den Ursprüngen und nach dem Umgang mit den erzielten Profiten (oder den erlittenen Verlusten). Die Unternehmensgeschichte eröffnete seit den 1920er Jahren (in Harvard) eine Debatte über die Möglichkeiten und die Einwirkungschancen einer Geschäftsethik, das heißt der Beziehungen zwischen Betriebsführung und Moral. ${ }^{51}$ Sie zeichnet die fluktuierenden Konturen eines Kapitalismus nach, der zugleich dezentralisiert und zentralisiert ist, oder doch fest und flüssig: fest, da er auf Institutionen, Regeln, Machtverhältnissen und beruflichen Milieus beruht, und flüssig, weil der Anteil der Fließbewegungen und der Mobilität eine Wachstumstendenz zeigt. ${ }^{52}$

Eine solche Geschichte sieht sich mit zwei Problemen konfrontiert: dem der Währung auf der einen Seite und dem der Ressourcen des Planeten auf der anderen Seite. Die Ökonomen, besonders die Regulationsschule, betonen die Bedeutung der Währung, der »ersten grundlegenden Institution einer Marktwirtschaft « - und damit des Kapitalismus - , der Währung als einem der Schlüssel einer »vollständigen Dezentralisierung der Austauschbeziehungen«. Sie heben gleichzeitig die Bedeutung des Währungsregimes hervor, also der Gesamtheit der Regeln, welche die Verwaltung des Systems der Zahlungen und der Kredite regulieren, die von den netzwerkartig organisierten Banken und von den Zentralbanken vergeben werden, welche die Rolle eines Kreditgebers letzter Instanz spielen. ${ }^{53}$ Sie sprechen dabei nun die Versuchung an, die durch die leichte Verschuldungsmöglichkeit für einen Teil der Staaten, der Haushalte und der Unternehmen entsteht, die wachsende Macht großer Finanzakteure und gleichzeitig ihre Verwundbarkeit angesichts von Finanz- und Wechselkrisen, deren Auswirkung nicht vorübergehend sondern tiefgreifend ist. Die Unternehmenshistoriker untersuchen ihrerseits die Fähigkeit eines Teils der Unternehmen, die Umwelt zu schädigen und zum Klimawandel beizutragen. Sie zeigen die Frühzeitigkeit und die Beharrlichkeit der Reaktionen der Anwohner ${ }^{54}$ und die Tatsache, dass unter gesellschaftlichem Druck ein Teil der Firmen heute dazu gebracht wird, sich für 
Produktformen oder Dienstleistungen zu öffnen, die den Erfordernissen einer nachhaltigen Entwicklung besser entsprechen. Die Unternehmensgeschichte beschäftigt sich also letztlich mit Fragestellungen bezüglich des Aufkommens eines durchführbaren Regulationsmodus, mit Kompromissen, die sie begründen können und mit den Bedingungen ihrer Erschöpfung oder ihrer Erneuerung.

\section{BIBLIOGRAPHIE}

Abend, Gabriel (2014): The Moral Background: An Enquiry into the History of Business Ethics, Princeton: Princeton University Press.

Amatori, Franco / Bigatti, Giorgio (2003): „Business History in Italy at the Turn of the Century«, in: Amatori, Franco / Jones, Geoffrey (Hg.): Business History around the World, Cambridge: Cambridge University Press, S. 215-222.

Amatori, Franco / Jones, Geoffrey (Hg.) (2003): Business History around the World, Cambridge: Cambridge University Press.

Anteby, Michel (2015): Manufacturing Morals: The Values of Silence in Business School Education, Chicago: University of Chicago Press.

Arena, Lise / Dang, Rani J. (2011): »À propos du développement de deux business schools d'élite au Royaume-Uni: une comparaison entre Cambridge et Oxford (1900-2000)«, Entreprises et Histoire, 65, Dezember, S. 60-82.

Barral, Stéphanie / Béaur, Gérard / Lambert, Christiane / Rémy, Jacques (2017): „L'agriculture et le capitalisme«, Entreprises et Histoire, 88, September, S. 166-181.

Bloch, Marc (1931): „Culture historique et action économique: à propos de l'exemple américain«, Annales d'histoire économique et sociale, 3 (9), 15. Januar, S. 1-4.

Bloch, Marc (1995): Histoire et historiens, Paris: Armand Colin.

Bloch, Marc / Hauser, Henri / Usher, Abbot Payton (1929a): "L'histoire économique aux États-Unis«, Annales d'histoire économique et sociale, 1 (2), S. 236-240.

Bloch, Marc / Hauser, Henri / Usher, Abbot Payton (1929b): »Nouvelles scientifiques«, Annales d'histoire économique et sociale, 1 (3), S. 417.

Bloch, Marc / Hauser, Henri / Usher, Abbot Payton (1930): „Encore des archives italiennes à Boston«, Annales d'histoire économique et sociale, 2 (5), S. 93.

Boothman, Barry E. C. (2001): »A Theme Worthy of Epic Treatment: N.S.B. Gras and the Emergence of American Business History«, Journal of Macromarketing, 21 (1), Juni, S. 61-73. Bouvier, Jean / Furet, François / Gillet, Marcel (1965): Le mouvement du profit en France au XIX ${ }^{e}$ siècle. Matériaux et études, Paris / Den Haag: Mouton.

Boyer, Robert (2015): Économie politique des capitalismes. Théorie de la régulation et des crises, Paris: La Découverte. 
Cailluet, Ludovic / Lemarchand, Yannick (2013): »L'École d'Orvault?«, in: Cailluet, Ludovic / Lemarchand, Yannick / Chessel, Marie-Emmanuelle (Hg.): Histoire et sciences de gestion, Paris: Vuibert, S. 1-13.

Cottereau, Alain (2006): »Sens du juste et usages du droit du travail: une évolution contrastée entre la France et la Grande-Bretagne au XIX ${ }^{\mathrm{e}}$ siècle«, Revue d'histoire du XIX ${ }^{e}$ siècle, 33,

2. Semester, S. 101-120.

Daumas, Jean-Claude (2018): La révolution matérielle. Une histoire de la consommation en France, XIX ${ }^{e}$ XXI siècle, Paris : Flammarion.

Duhamel, Georges (1930): Scènes de la vie future, Paris: Mercure de France.

Dumoulin, Olivier (2003): Le rôle social de l'historien, Paris: Albin Michel.

Duranton-Crabol, Anne-Marie (2001) »De l'anti-américanisme en France vers 1930: la réception des Scènes de la vie future«, Revue d'histoire moderne et contemporaine, März, S. 120-137.

Febvre, Lucien (1931): »Un exemple et une leçon«, Annales d'histoire économique et sociale, 3 (11), S. 367-368.

Fink, Carole (1997): Marc Bloch: une vie au service de l'histoire, Lyon: Presses Universitaires de Lyon.

Fridenson, Patrick / Paquy, Lucie (2008): »Du haut enseignement commercial à l'enseignement supérieur de gestion (XIX ${ }^{\mathrm{e}}-\mathrm{XX}{ }^{\mathrm{e}}$ siècles)«, in: Lenormand, Paul (Hg.): La Chambre de commerce et d'industrie de Paris 1803-2003. II. Études thématiques, Genf: Droz, S. 211-212.

Friedmann, Georges (1937): „Bat'a ou les leçons d'une entreprise géante«, Annales d'histoire économique et sociale, 9 (47), S. 501-507.

Galambos, Louis (1967): American Business History, Washington: Service Center for Teachers of History.

Gemelli, Giuliana (1985): Fernand Braudel, Paris: Odile Jacob.

Gille, Bertrand (1958) : »Note liminaire«, Histoire des entreprises, 1, Mai, S. 6.

Gille, Bertrand (1963): »Liminaire«, Histoire des entreprises, 12, November (tatsächlich 1965), S. 7.

Gras, Norman S. B. (1931): »Les affaires et l'histoire des affaires«, Annales d'histoire économique et sociale, 3 (9), 15. Januar, S. 5-10.

Gras, Norman S. B. (1950): »Past, Present and Future of the Business Historical Society«, Bulletin of the Business Historical Society, 24 (1), März, S. 1-12.

Hachez-Leroy, Florence (1999): L'Aluminium français. L'invention d'un marché, 1911-1983, Paris: CNRS Editions.

Harvey, John L. (2004): "An American Annales? The AHA and the Revue internationale d'histoire économique of Lucien Febvre and Marc Bloch«, Journal of Modern History, 76, September, S. 528-621. Hauser, Henri (1927): Les débuts du capitalisme, Paris: Alcan.

Hendley, Brian (2000): »Whitehead and Business Education: A Second Look«, Interchange, 31 (2-3), Juni, S. 179-195.

Hughes, Jonathan R. T. (1983): »Arthur Cole and Entrepreneurial History«, Business and Economic History, Zweite Serie, 12, S. 133-144.

Huvos, Kornel (1972): Cinq mirages américains. Les États-Unis dans l'œuvre de Georges Duhamel, Jules Romains, André Maurois, Jacques Maritain et Simone de Beauvoir, Paris: Marcel Didier. 
Jones Geoffrey / Sluyterman, Keetie E. (2003): »British and Dutch Business History«, in: Amatori, Franco / Jones, Geoffrey (Hg.): Business History around the World, Cambridge: Cambridge University Press, S. 114-115.

Judges A. V. (1935): »Pour la conservation et l'utilisation des archives d'entreprises en GrandeBretagne«, Annales d'histoire économique et sociale, 7 (35), S. 448-450.

Kocka, Jürgen (2017): Geschichte des Kapitalismus, 3. überarbeitete Aufl., München: C. H. Beck. Lamoreaux, Naomi / Raff, Daniel / Temin, Peter (2003): »Beyond Markets and Hierarchies: Toward a New Synthesis of American Business History«, The American Historical Review, 108 (2), 1. April, S. 404-433.

Lefebvre, Philippe (2003): L'invention de la grande entreprise. Travail, hiérarchie, marché. France, fin $X V I I{ }^{e}$-début $\mathrm{XX}^{e}$ siècle, Paris : PUF.

Lemercier, Claire (2014): »Naissance de l'entreprise et formes antérieures d'organisation «, in: Segrestin, Blanche / Roger, Baudoin / Vernac, Stéphane (Hg.): L'entreprise point aveugle du savoir, Auxerre: Editions Sciences Humaines, S. 153-163.

Leuilliot, Paul (1931): „Les archives d'une banque contemporaine: ce qu'elles contiennent, ce que l'on peut en tirer«, Annales d'histoire économique et sociale, 3 (11), S. 368-378.

Leuilliot, Paul (1939): »État et ententes d'entreprises«, Annales d'histoire sociale, 1 (3), Juni, S. 295297.

Lincoln, Andrew (1981): »Le syndicalisme patronal à Paris de 1815 à 1848: une étape de la formation d'une classe patronale«, Le Mouvement Social, 161, Januar-März, S. 11-34.

Lipartito, Kenneth (2016): »Reassembling the Economic: New Departures in Historical Materialism«, The American Historical Review, 121, Februar, S. 101-139.

Massard-Guilbaud, Geneviève (2010): Histoire de la pollution industrielle. France, 1789-1914, Paris: Éditions de l'EHESS.

Minkes, Leonard (2011): »Early years in University development in management education: reflections and reminiscences on the University of Birmingham (1950s-1970s) «, Entreprises et Histoire, 65, Dezember, S. 83-95.

O'Connor, Ellen S. (2012): Creating New Knowledge in Management: Appropriating the Field's Lost Foundations, Stanford: Stanford University Press.

Omnès, Cécile (2007): La gestion du personnel au Crédit lyonnais de 1863 à 1939: une fonction en devenir (genèse, maturation et rationalisation), Brüssel: Peter Lang.

Palmade, Guy P. (1962): Capitalisme et capitalistes au XIX'e siècle, Paris: A. Colin.

Paxton, Robert O. (1977) : "L'affaire des carbures et l'abolition du délit de coalition, 1915-1926«, in: Fridenson, Patrick (Hg.): 1914-1918, l'autre front, Paris: Éditions ouvrières, S. 145-170.

Sabel, Charles F. / Zeitlin, Jonathan (Hg.) (1997): World of Possibilities: Flexibility and Mass Production in Western Industrialization, Cambridge: Cambridge University Press / Paris: Éditions de la MSH.

Sass, Stephen A. (1986): Entrepreneurial Historians and History: Leadership and Rationality in American Economic Historiography, 1940-1960, New York: Garland.

Schmidt, Charles (1930): „L'organisation rationnelle des entreprises: les archives privées et l'histoire«, Annales d'histoire économique et sociale, 2 (5), S. 64-66. 
Schröter, Harm (2003): „Business History in German-Speaking States«, in: Amatori, Franco / Jones, Geoffrey (Hg.): Business History around the World, Cambridge: Cambridge University Press, S. $173-182$.

Scranton, Philip (1997): Endless Novelty. Specialty Production and American Industrialization, 1865-1925, Princeton: Princeton University Press.

Scranton, Philip / Fridenson, Patrick (2013): Reimagining Business History, Baltimore: Johns Hopkins University Press.

Sicilia, David B. (1995): »Cochran's Legacy: A Cultural Path Not Taken«, Business and Economic History, Zweite Serie, 24 (1), S. 27-39.

Smith, Michael S. (2006): The Emergence of Modern Business Enterprise in France, 1800-1930, Cambridge (Mass.): Harvard University Press.

Sombart, Werner (1902): Der moderne Kapitalismus, Leipzig: Duncker \& Humblot.

Torres, Félix (1995): »Histoire et mémoire de l'entreprise«, Communication et Organisation, 7, https://journals.openedition.org/communicationorganisation/1775 (eingesehen am 22.11.2018)

Verley, Patrick (1997): L'échelle du monde. Essai sur l'industrialisation de l'Occident, Paris: Gallimard.

Wee, Herman van der (2006): « Henri Hauser et l'histoire du capitalisme du XVI ${ }^{\mathrm{e}}$ au XVIII siècle ", in : Soutou, Georges-Henri / Marin, Séverine-Antigone (Hg.): Henri Hauser 1866-1946. Humaniste, historien, républicain, Paris: Presses de l'Université Paris-Sorbonne, S. 119-128.

Williams, Daryl B. (2009): »The Historiography of Business History«, April, http:// daryl.williams.tripod.com/index1.html (eingesehen am 1. März 2013).

\section{NOTES}

1. Palmade (1962), S. 10-11. „Capitalisme«, in: Trésor de la langue française, 1994. Kocka, S. 7-8.

2. Sombart (1902).

3. Hauser (1927). Siehe Wee (2006).

4. Gras (1950).

5. Hendley (2000). Ich danke Martin Giraudeau für diesen Hinweis.

6. Boothman (2001).

7. Galambos (1967). Williams (2009).

8. Harvey (2004), besonders S. 597-598.

9. Gras (1931).

10. Cailluet / Lemarchand (2013), S. 2-3.

11. Bloch (1931).

12. Bloch (1995), S. 25-28.

13. Französische Übersetzung der Biographie: Fink (1997), S. 111 u. S. 132. Erwähnung des Unternehmensberaters: Torres (1995). Publikation der Habilitationsschrift: Dumoulin (2003), S. 266.

14. Duhamel (1930). Vgl. Huvos (1972) und Duranton-Crabol (2001) (die den Artikel M. Blochs nicht berücksichtigt).

15. Dumoulin (2003), S. 265. Wir halten fest, dass der Ausdruck »Ereignisse« (événements) 1931 nicht zum Vokabular Marc Blochs gehörte.

16. Fridenson / Paquy (2008).

17. Schmidt (1930).

18. Siehe hier die vervollständigten Belege: Bloch / Hauser / Usher (1929a), (1929b), (1930). 
19. Febvre (1931).

20. Judges A. V. (1935).

21. Leuilliot (1931), (1939).

22. Friedmann (1937).

23. Für einen detaillierten Überblick, siehe Amatori / Jones (2003).

24. Galambos (1967), S. 20-24. Hughes (1983). Und vor allem Sass (1986).

25. Sicilia (1995).

26. Scranton (1997). Sabel / Zeitlin (1997).

27. Lamoreaux / Raff / Temin (2003).

28. Arena / Dang (2011). Minkes (2011).

29. Jones / Sluyterman (2003).

30. Schröter (2003).

31. Internetseiten der Éditions de l'EHESS, eingesehen am 30. September 2013.

32. Bouvier / Furet / Gillet (1965).

33. Archives Nationales du Monde du Travail, 182 AQ 4 und 19, Papiere Bertrand Gille.

34. Gespräch mit Didier Ozanam, ehemaliger Generalsekretär des Centre de Recherches Historiques,

17. Juni 2015.

35. Gille (1958).

36. Gemelli (1985), S. 285-286.

37. Gille (1963). Diese Ausgabe publizierte das vollständige Inhaltsverzeichnis der zwölf Nummern.

38. Gemelli (1985), S. 286-288.

39. Amatori / Bigatti (2003).

40. Verley (1997).

41. Lemercier (2014), S. 156.

42. Smith (2006).

43. Lincoln (1981).

44. Paxton (1977). Hachez-Leroy (1999).

45. Barral et al. (2017).

46. Cottereau (2006).

47. Lefebvre (2003).

48. Omnès (2007).

49. Daumas (2018).

50. Lipartito (2016).

51. Siehe O'Connor (2012); Anteby (2015); zu einer Geschichte der Geschäftsethik in den USA der 1850 er bis 1930er Jahre: Abend (2014).

Für einen (nicht erschöpfenden) Überblick zur Präsenz der Geschichte in den höheren Ausbildungsgängen und den Forschungsprogrammen der Betriebswissenschaft in Frankreich, siehe Cailluet / Lemarchand (2013), S. 9-10.

52. Scranton / Fridenson (2013).

53. Boyer (2015), S. 22-23 und 316-318.

54. Massard-Guilbaud (2010). 
INDEX

Mots-clés : capitalisme, business history, histoire des entreprises

Schlüsselwörter : Kapitalismus, business history, Unternehmensgeschichte

\section{AUTEURS}

\section{PATRICK FRIDENSON}

Patrick Fridenson ist directeur d'études am Centre de recherches historiques (CRH) der École des hautes études en sciences sociales (EHESS). Nähere Informationen finden Sie hier. 